\title{
Screening for Macroprolactinemia Is Indispensable for Proper Treatment of Hyperprolactinemia Microadenoma
}

\section{JING DU}

Zhejiang Provincial People's Hospital https://orcid.org/0000-0001-7519-8531

\section{Yanchun Li}

Zhejiang Chinese Medical University

\section{Yuhuan Shen}

Guangdong Provincial People's Hospital

\section{Wei Yang}

Zhejiang Provincial People's Hospital

\section{Ying Ye}

zhoushan dinghai guanghua hospital

Yongjian Chen ( $\nabla$ lab_cyj@126.com)

Zhejiang Provincial People's Hospital

\section{Research article}

Keywords: Macroprolactinaemia, Hyperprolactinemia, Prolactin microadenoma, Macroprolactin microadenoma

Posted Date: December 28th, 2020

DOI: https://doi.org/10.21203/rs.3.rs-133454/v1

License: (c) (1) This work is licensed under a Creative Commons Attribution 4.0 International License. Read Full License 


\section{Abstract}

BACKGROUND: Macroprolactin microadenoma is a combination of non-functional microadenomas with macroprolactinemia. This study aimed to explore the significance of macroprolactinemia screening for the antidiastole of prolactin microadenoma and macroprolactin microadenoma.

METHODS: Retrospective analysis of patients with pituitary microadenoma and screening for macroprolactinemia in patients with hyperprolactinemia was conducted. Based on the prolactin content and screening results, patients were divided into non-functional microadenoma, prolactin microadenoma, and macroprolactin microadenoma groups. The existing forms of prolactin in serum samples were analyzed by gel filtration chromatography and a luminescence immunoassay analyzer. The clinical course of patients and treatment were retrospectively reviewed.

RESULTS: The results of gel filtration chromatography confirmed that prolactin in patients with macroprolactin microadenoma was mainly in the form of macromolecules, and the small molecular prolactin was within the normal range. Among 84 patients with hyperprolactin microadenomas, 9 (10.7 $\%)$ were diagnosed with macroprolactin microadenoma, and 5 (55.6\%) were treated with bromocriptine. The prolactin content $(55.9 \sim 81.0 \mathrm{ng} / \mathrm{mL})$ in the macroprolactin microadenoma group was elevated before the screening and significantly decreased $(11.2 \sim 21.2 \mathrm{ng} / \mathrm{mL})$ after screening. The incidence of clinical manifestations was the same as that of the non-functional microadenoma group, but the rate of drug use was higher, which was similar to that of the prolactin microadenoma group.

CONCLUSION: Macroprolactinemia screening can effectively identify prolactin microadenomas and macroprolactin microadenomas, which prevent misdiagnosis and mistreatment of macroprolactin microadenomas.

\section{Background}

Based on clinical manifestations and endocrine hormone levels, pituitary adenomas are classified as non-functional adenomas, prolactinomas, growth hormone-secreting adenomas, adrenocorticotropic hormone-secreting adenomas, etc. According to the size of the tumor, pituitary adenomas $<10 \mathrm{~mm}$ in diameter are named microadenomas. The two main forms of microadenomas are prolactin microadenoma and non-functional microadenoma; both have similar imaging features, but different hormone (prolactin) levels. Excessive secretion of prolactin in patients with prolactin microadenoma leads to hyperprolactinemia, which often causes symptoms, such as menstrual disorders, amenorrhea, galactorrhea, and erectile dysfunction. Dopamine agonists are the first choice for treatment $[1,2]$. Although there is no excessive secretion of prolactin in patients with non-functional microadenoma, they may have the symptoms mentioned above caused by compression of the pituitary [3]. If so, follow-up monitoring of prolactin may provide critical information for treatment $[4,5]$. Therefore, emerging hyperprolactinemia is the key to distinguish prolactin microadenoma and non-functional microadenoma. However, macroprolactinemia, reported in recent years, is a pseudohyperprolactinemia caused by the 
accumulation of macromolecule prolactin, which is formed in the blood circulation when prolactin is combined with autoantibodies, resulting in an accumulation in the circulation [6, 7]. If a non-functional microadenoma is accompanied by macroprolactinemia (hereinafter referred to as macroprolactin microadenoma), it could easily be misdiagnosed as prolactin microadenoma. In this study, we conducted macroprolactinemia screening in patients with hyperprolactinemia microadenoma and evaluated the value of macroprolactinemia screening in the differential diagnosis of macroprolactin microadenoma and prolactin microadenoma, thus avoiding misdiagnosis and mistreatment of macroprolactin microadenoma.

\section{Methods}

\section{Patients}

A total of 108 patients were included in this retrospective study according to the following inclusion criteria: female sex, age ranging from 18 to 50 years, visit to the Zhejiang Provincial People's Hospital between January 2014 and March 2019, diagnosis of pituitary microadenoma (diameter of adenoma < $10 \mathrm{~mm}$ ) during the first magnetic resonance dynamic enhancement examination, simultaneously diagnosis of hyperprolactinemia (prolactin $>50 \mathrm{ng} / \mathrm{mL}$ ) before macroprolactinemia screening (84 patients), and microadenoma with prolactin content within the reference range (prolactin $<25 \mathrm{ng} / \mathrm{mL}$ ) in non-treated conditions (24 patients). Exclusion criteria included pregnancy, menopause, thyroid disease, growth hormone adenoma, renal adrenal adenoma, and depression [5]. The medical history and related symptoms of the included cases were recorded. This study was approved by the Ethics Committee of Zhejiang Provincial People's Hospital.

\section{Prolactin content assay}

The I2000SR chemiluminescence immunoassay analyzer (Abbott Laboratories, Chicago, IL, USA) and its supporting kit (Lot: 91424UI00, Abbott Ireland Diagnostics Division, Lisnamuck, Longford, Ireland) were used to test the prolactin levels in serum samples. For gel filtration chromatography elution, the prolactin level was analyzed using several other luminescence immunoassay analyzers, such as Immulite2000 luminescence immunoassay analyzer (Siemens Healthcare Diagnostic Inc., New Jersey, USA) and its supporting kit (Lot: 343, Glyn Rhonwy, Lianberis, United Kingdom), E602 luminescence immunoassay analyzer (Roche Diagnostics GmbH, 882 Ichige, JAPAN) and the kit (Lot: 33441703, Sandhofer Strasse 116, GERMANY), ACCESS2 luminescence immunoassay analyzer (Beckman Coulter Inc., Lake Hazeltine Drive Chaska, USA) and the kit (Lot: 724837, Lake Hazeltine Drive Chaska, USA).

\section{Macroprolactinemia screening and diagnosis}

Polyethylene glycol (PEG) precipitation was used for the screening of macroprolactinemia in hyperprolactinemia samples [8,9]. Briefly, $200 \mu \mathrm{L}$ of serum samples were mixed with an equal amount of 25\% PEG6000 (Sigma, St. Louis, MO, USA), set aside at room temperature for $10 \mathrm{~min}$, centrifuged at $1,800 \mathrm{~g}$ for $10 \mathrm{~min}$, and the supernatant was assayed on an I2000SR chemiluminescence immunoassay 
analyzer. The recovery rate of prolactin was calculated as follows: recovery rate $=$ (prolactin content in the supernatant after PEG precipitation $\times 2) \times 100 \%$ / prolactin content before PEG precipitation. A recovery rate of prolactin $<50 \%$, means that the prolactin in serum is mainly macromolecular prolactin, and this is judged as macroprolactinemia. Conversely, if the recovery rate is $\geq 50 \%$, the prolactin in serum is primarily the type of small-molecule prolactin and it is true hyperprolactinemia.

\section{Case grouping}

All included cases were divided into three groups according to the results of prolactin testing and macroprolactinemia screening. In total, 75 patients with microadenomas were screened for true hyperprolactinemia and classified as prolactin microadenomas, with an average age of $35.0 \pm 9.3$ years. Nine patients with a mean age of $29.0 \pm 9.6$ years were screened for macroprolactinemia and classified as macroprolactin microadenomas. Twenty-four patients with an average age of $36.3 \pm 10.3$ years were classified as non-functional microadenomas because the concentration of prolactin was within the reference range in the non-treated state. There was no significant difference in age distribution among the three groups.

\section{Gel filtration chromatography analysis of prolactin}

Serum samples from patients with prolactin microadenomas and macroprolactin microadenomas were separated by gel filtration chromatography (HiLoad 16/600 Superdex 75 pg, GE Life Sciences, USA). The chromatographic elution was collected at $1.0 \mathrm{~mL} /$ tube, and the forms of prolactin in the chromatographic solution were analyzed using a luminescence immunoassay analyzer.

\section{Follow-up}

The patients diagnosed with macroprolactin microadenomas were followed up by telephone, and their clinical situations or symptoms of menstrual disorders, amenorrhea, galactorrhea, and medication were recorded.

\section{Statistics}

The date of prolactin concentration was non-normally distributed and was shown as median (quartile). Statistical analyses were performed using SPSS 17.0. The statistical significance between groups was determined by the Mann-Whitney test. The incidence of symptoms, such as galactorrhea, between groups was compared using the chi-square $(\chi 2)$ test. $P<0.05$ was considered statistically significant.

\section{Results}

\section{Detection of macroprolactin microadenomas}

The magnetic resonance images of pituitary microadenomas were indistinguishable between prolactin microadenoma (sample A) and macroprolactin microadenoma (sample B), as shown in Fig. 1. Gel filtration chromatography and an I2000SR luminescence analyzer were combined to determine the form 
of prolactin in the serum of patients with hyperprolactinemia microadenomas. The results showed that the prominent peak representing small-molecule prolactin was present in the prolactin microadenoma group, whereas the main peak representing macromolecular prolactin was in the macroprolactin microadenoma group (Fig. 2A). The concentrations of macromolecular or small-molecule prolactin were calculated by the area under the curve, which was $58.9 \mathrm{ng} / \mathrm{mL}$ and $19.1 \mathrm{ng} / \mathrm{mL}$, respectively. The samples from the macroprolactin microadenoma group were also detected by three other illuminating analyzers (immulite2000, E 602, and Access2), and the detection results were identical to those of I2000SR (Fig. 2B). After gel filtration chromatography screening for macroprolactinemia, 9 of $84(10.7 \%)$ hyperprolactinemia microadenoma patients were diagnosed with macroprolactin microadenomas.

\section{Prolactin concentration is within the normal range in patients with macroprolactin microadenomas.}

The concentration of prolactin in the serum was detected before and after macroprolactinemia screening. The results in Fig. 3 show that prolactin content were $14.1(9.9 \sim 20.6) \mathrm{ng} / \mathrm{mL}, 60.0(55.9 \sim 81.0) \mathrm{ng} / \mathrm{mL}$, and $86.1(65.5 \sim 128.0) \mathrm{ng} / \mathrm{mL}$, in non-functional microadenomas, macroprolactin microadenomas and prolactin microadenomas, respectively, before the screening. There was a statistical difference in these three groups, especially between the two groups of macroprolactin microadenomas and non-functional microadenomas $(P<0.001)$. After macroprolactinemia screening, the prolactin content in the macroprolactin microadenoma group decreased to $15.0(11.2 \sim 21.2) \mathrm{ng} / \mathrm{mL}$, which was similar to that of the non-functional microadenoma group, and the difference was not significant $(P>0.05)$.

\section{Follow-up of the clinical information}

We retrospectively reviewed the clinical data of the included patients. The details of symptoms and medication of the cases are shown in Table 1. Analytically, the incidence of menstrual disorders, amenorrhea, and galactorrhea were not statistically significantly different between the non-functional microadenomas and the macroprolactin microadenomas $(P>0.05)$. The incidence of menstrual disorders and galactorrhea in the prolactin microadenoma group were $38.7 \%$ and $28.0 \%$, respectively, which were higher than those in the non-functional microadenomas ( $16.7 \%$ and $0.0 \%$, respectively). The difference was statistically significant $(P<0.05)$. The usage rate of bromocriptine in macroprolactin microadenomas and prolactin microadenomas was $55.6 \%$ and $38.7 \%$, respectively. There was no significant difference between the two groups $(P>0.05)$, but they were both higher than that in nonfunctional microadenoma group $(P<0.01)$.

\section{Manifestations of patients with macroprolactin microadenomas}

The details were reviewed in patients with macroprolactin microadenomas. Before macroprolactinemia screening, 5 out of 9 (55.6\%) patients with macroprolactin microadenomas received bromointine treatment for 1-4 years, respectively, with no significant difference in tumor size, which was unchanged 
in 3 patients and even slightly larger in 2 . The other 4 patients were not treated with bromocriptine. During the follow-up in March 2019, all 9 patients had no symptoms of galactorrhea, menstrual disorders, or other relevant symptoms.

\section{Discussion}

Prolactinoma is characterized by excessive secretion of prolactin from the pituitary gland, accounting for one-half of pituitary adenomas, $80 \%$ of which are microadenomas [10]. Non-functional adenomas account for one-third of pituitary adenomas, and the vast majority of them do not cause clinical manifestations due to the lack of excessive hormone secretion and their small size. Non-functional microadenomas often exhibit no clinical symptoms, and their diagnosis needs to entail the evaluation of multiple hormone levels and the making of an exclusion diagnosis [11,5]. For patients with a specific imaging diagnosis of microadenomas, clinical manifestations combined with laboratory tests, such as growth hormone, insulin-like growth factor 1, adrenocorticotropic hormone, cortisol, and thyroidstimulating hormone, can effectively exclude growth hormone adenoma, adrenal hormone adenoma, and thyroid-stimulating hormone adenoma. The remaining prolactin microadenomas and non-functional microadenomas are distinguished by examining the excessive secretion of prolactin in the pituitary gland. Excluding some physiological or pathological conditions, such as pregnancy, thyroid disorders, and antidepressants, hyperprolactinemia can effectively manifest as excessive secretion of pituitary prolactin [2]. However, macroprolactinemia is a pseudohyperprolactinemia caused by the accumulation of macromolecular prolactin, mainly the complex of prolactin and its autoantibody, in the circulation, which is not an excessive secretion of pituitary prolactin [12, 7]. Therefore, neglecting the existence of macroprolactinemia can lead to the misdiagnosis of non-functional microadenomas as prolactin microadenomas and even result in the wrong treatments.

Through gel filtration chromatography and prolactin luminescence detection, we confirmed that prolactin in macroprolactin microadenoma samples was mainly in the form of macromolecules, and there was no accumulation of small molecule prolactin, which confirmed that this was a non-functional microadenoma combined with the presence of macroprolactinemia. The presence of macroprolactin microadenomas indicated that we should not make a diagnosis of prolactin microadenomas for patients with clear imaging diagnosis of microadenoma and hyperprolactinemia unless the macroprolactinemia screening is accomplished. The macroprolactinemia screening could prevent non-functional microadenomas from being misdiagnosed as prolactin microadenomas. In addition, several chemiluminescence immunoassay analyzers were used to detect the value of prolactin in the chromatographic elution from the sample of macroprolactin microadenomas. These results showed that various commercial prolactin detection reagents, including Roche's second-generation prolactin testing reagent, have high cross-reactivity with macroprolactin. Therefore, screening for macroprolactinemia in hyperprolactinemia cases is indispensable and universal, regardless of the detection reagents and instruments used. 
In this study, we identified 9 patients (10.7\%) with macroprolactin microadenomas among 84 patients with hyperprolactinemia microadenomas, which is lower than the prevalence rate $(15 \%-46 \%)$ of macroprolactinemia in hyperprolactinemia $[13,14]$ and higher than the incidence $(3.5 \%)$ of macroprolactinemia in prolactinomas [15]. During the follow-up of patients with macroprolactin microadenoma: (1) the 5 patients receiving bromocriptine treatment showed no change in tumor size and had no corresponding clinical symptoms as the 4 patients without bromocriptine treatment, indicating that bromocriptine therapy was not only ineffective, but also unnecessary for patients with macroprolactin microadenomas. (2) The results in Table 1 suggest that there was no statistically significant difference in the related symptoms between the two groups of macroprolactin microadenomas and non-functional microadenomas. (3) After screening for macroprolactinemia, the concentration of prolactin in patients with macroprolactin microadenomas was within the normal range and similar to that in patients with non-functional microadenomas. These data fully demonstrate that the nature of macroprolactin microadenoma is actually non-functional microadenoma [5]. Moreover, there are few or no associated symptoms in macroprolactin microadenomas in the absence of biological activity of macroprolactin in the body. Menstrual disorders, in some cases, may be related to other factors besides the pituitary, which is consistent with related reports [16].

Although the macroprolactin microadenomas are actually non-functional microadenomas, the prolactin concentration within them is moderately elevated $(50 \sim 150 \mathrm{ng} / \mathrm{mL})$ before macroprolactin screening (Figure. 3C). Due to the absence of screening for macroprolactinemia, some patients with macroprolactin microadenomas were mistakenly treated with bromocriptine. The rate of drug use reached $55.6 \%$, which was significantly higher than that of non-functional microadenomas $(0.0 \%, P<0.05)$. However, there was no significant difference in the rate of drug use between the macroprolactin microadenomas and prolactin microadenomas $(38.7 \%, P>0.05)$.

\section{Conclusion}

Macroprolactin microadenomas are non-functional microadenomas accompanied by macroprolactinemia. In addition, there is a high proportion of macroprolactin microadenomas in patients with hyperprolactinemia microadenomas. Without screening for macroprolactinemia, more patients with macroprolactin microadenomas could be misdiagnosed as prolactin microadenomas, and receive unnecessary treatment.

\section{Declarations}

\section{Ethics approval and consent to participate}

This study was approved by the Ethics Committee of Zhejiang Provincial People's Hospital. Informed consent was not required owing to the retrospective design.

\section{Consent for publication}


Not applicable.

\section{Availability of data and materials}

The raw data in the current study may be made available upon reasonable request from the corresponding authors.

\section{Competing interests}

The authors declare no conflict of interest. None of the contents of this manuscript has been previously published or is under consideration elsewhere.

\section{Funding}

This research was supported by Zhejiang Public Welfare Technology Application Research Project (No. LGF19H080006, 2017C33091), Medical and Health Science and Technology Project of Zhejiang Province (No.2019RC014, 2019RC115, 2019KY016, 2017KY006, 2017KY209), Zhejiang Students' Science and Technology Innovation Activity Plan (No. 2019R410053).

\section{Authors' contributions}

Yongjian Chen: Conceptualization, Supervision, Methodology.

Jing Du: Validation, Writing - Original Draft, Software.

Jing Du, Yanchun Li, Yuhuan Shen, Wei Yang, Ying Ye: Project administration, Writing - Review \& Editing.

Yuhuan Shen: Visualization, Data Curation.

All authors read and approved the manuscript.

\section{Acknowledgements}

Not applicable.

\section{References}

1. Melmed S, FF C, AR H, et al. Diagnosis and treatment of hyperprolactinemia: an Endocrine Society clinical practice guideline. J Clin Endocrinol Metab. 2011;96:273-88.

2. Faje A, Nachtigall L. Current treatment options for hyperprolactinemia. Expert Opin Pharmacother. 2013;14:1611-25.

3. Can M, Guven B, Atmaca H, Acıkgoz S, Mungan G. Clinical characterization of patients with macroprolactinemia and monomeric hyperprolactinemia. Kaohsiung J Med Sci. 2011;27:173-6. 
4. Chanson P, Raverot G, Castinetti F, Cortet-Rudelli C, Galland F, Salenave S. Management of clinically non-functioning pituitary adenoma. Ann Endocrinol (Paris). 2015;76:239-47.

5. Molitch ME. Diagnosis and Treatment of Pituitary Adenomas. Jama. 2017;317:516-24.

6. MN F-W, John R, AR E. Macroprolactin; high molecular mass forms of circulating prolactin. Ann Clin Biochem. 2005;42:175-92.

7. Hattori N, Ishihara T, Saiki Y. Macroprolactinaemia: prevalence and aetiologies in a large group of hospital workers. Clin Endocrinol (Oxf). 2009;71:702-8.

8. Fahie-Wilson M, TP S. Determination of prolactin: the macroprolactin problem. Best Pract Res Clin Endocrinol Metab. 2013;27:725-42.

9. YJ C, GZ S, ZN W. A new criteria for screening macroprolactinemia using polyethylene glycol treatment combined with different assays for prolactin. Eur Rev Med Pharmacol Sci. 2016;20:178894.

10. Fernandez A, Karavitaki N, JA W. Prevalence of pituitary adenomas: a community-based, crosssectional study in Banbury (Oxfordshire, UK). Clin Endocrinol (Oxf). 2010;72:377-82.

11. ME M. Pituitary tumours: pituitary incidentalomas. Best Pract Res Clin Endocrinol Metab. 2009;23:667-75.

12. Hattori N, Nakayama Y, Kitagawa K, Li T, Inagaki C. Development of anti-PRL (prolactin) autoantibodies by homologous PRL in rats: a model for macroprolactinemia. Endocrinology. 2007;148:2465-70.

13. Kasum M, Oreskovic S, Zec I, et al. Macroprolactinemia: new insights in hyperprolactinemia. Biochem medica. 2012;22:171-9.

14. Kasum M, Pavičić-Baldani D, Stanić $P$, et al. Importance of macroprolactinemia in hyperprolactinemia. Eur J Obstet Gynecol Reprod Biol. 2014;183:28-32.

15. Elenkova A, Genov N, Abadzhieva Z, et al. Macroprolactinemia in patients with prolactinomas: prevalence and clinical significance. Exp Clin Endocrinol Diabetes. 2013;121:201-5.

16. Thirunavakkarasu K, Dutta P, Sridhar S, et al. Macroprolactinemia in hyperprolactinemic infertile women. Endocrine. 2013;44:750-5.

\section{Table}


Table 1

The incidence of symptoms and drug use rates in patients with non-functional microadenomas, macroprolactin microadenomas and prolactin microadenomas

\begin{tabular}{|c|c|c|c|c|c|}
\hline \multirow[t]{2}{*}{ Group } & \multirow{2}{*}{$\begin{array}{l}\text { Number of } \\
\text { cases }\end{array}$} & \multicolumn{3}{|c|}{ Symptoms(cases (\%)) } & \multirow{2}{*}{$\begin{array}{l}\text { Drug use } \\
\text { cases(\%) }\end{array}$} \\
\hline & & Paramenia & Amenorrhea & Galactorrhea & \\
\hline $\begin{array}{l}\text { non-functional } \\
\text { microadenomas }\end{array}$ & 24 & $4(16.7)$ & $1(4.2)$ & $0(0.0)$ & $0(0.0)$ \\
\hline $\begin{array}{l}\text { macroprolactin } \\
\text { microadenomas }\end{array}$ & 9 & $4(44.4)$ & $0(0.0)$ & $0(0.0)$ & $5(55.6)^{\star \#}$ \\
\hline $\begin{array}{l}\text { prolactin } \\
\text { microadenomas }\end{array}$ & 75 & $29(38.7)^{\star}$ & $7(9.3)$ & $21(28.0)^{*}$ & $29(38.7)^{\star}$ \\
\hline
\end{tabular}

\section{Figures}

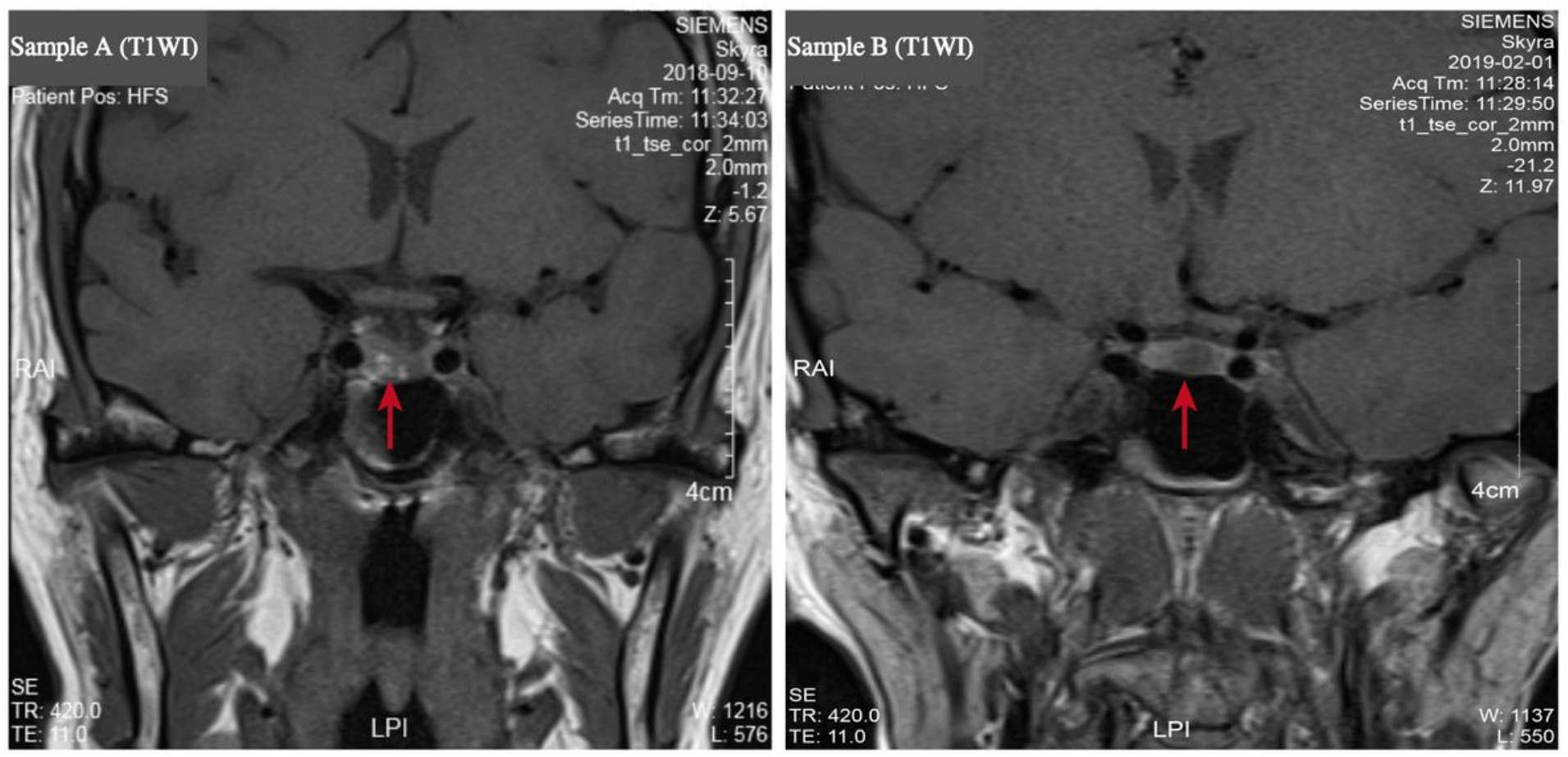

Figure 1

Magnetic resonance images of pituitary microadenomas. Microadenomas are indicated by red arrows; Left is the image of a patient with prolactin microadenoma, and the right is the image of macroprolactin microadenoma in T1WI. 

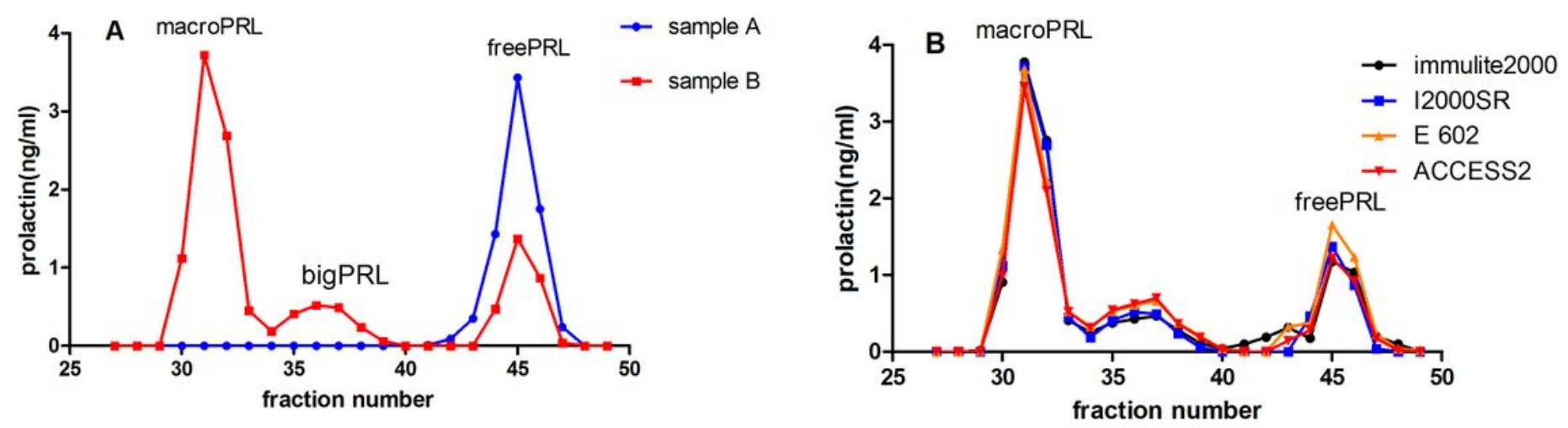

Figure 2

The existing forms of prolactin in prolactin microadenomas and macroprolactin microadenomas. A: The analysis curves of prolactin in sample A and sample B using an I2000SR luminescence analyzer. B: The analysis curves of prolactin in sample $B$ by four different luminescence analyzers. MacroPRL, bigPRL, and freePRL represent three molecular forms of prolactin: The macroprolactin molecule, the bimolecular form of prolactin, and the small molecule prolactin.
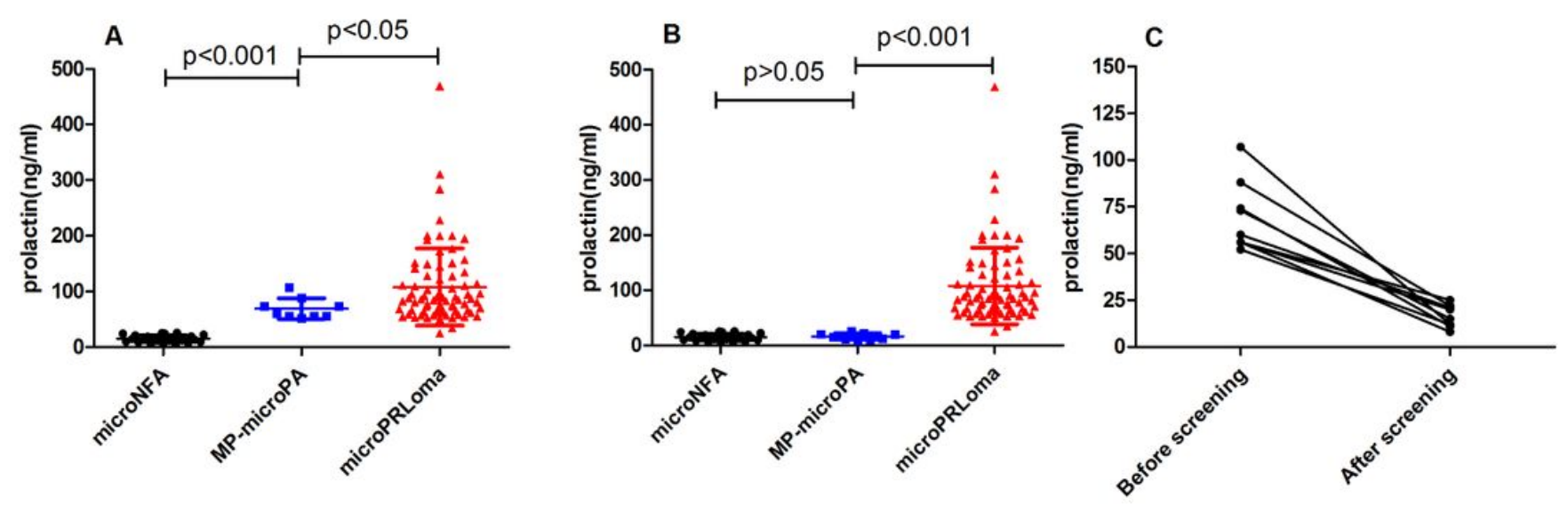

\section{Figure 3}

Comparison of the prolactin content in patients with non-functional microadenomas, macroprolactin microadenomas, and prolactin microadenomas. A: The prolactin concentration comparison before macroprolactinemia screening. B: The prolactin concentration comparison after macroprolactinemia screening. C: Changes in prolactin content before and after screening for patients with macroprolactin microadenomas. 